\title{
Ammar Ahmed Chaudhry: do the right thing for the right reasons!
}

Submitted Sep 24, 2018. Accepted for publication Oct 02, 2018.

doi: $10.21037 /$ jtd.2018.10.18

View this article at: http://dx.doi.org/10.21037/jtd.2018.10.18

\section{Editor's note}

Some people dream of success, while others get up every morning and make it happen. As a child, Prof. Ammar Ahmed Chaudhry had seen his parents struggle with complex medical conditions. Seeing his mother go through a series of complicated biopsies, he started out to pursue the possibility of "virtual biopsy", which is defined as biological profiles of specific tumors that may help predict a patient's response to treatment and probability of long-term survival, and decided to enter the field of diagnostic and interventional radiology.

While Prof. Chaudhry is striving hard day after day to turn "virtual biopsy" into reality, he has been gaining comprehensive exposure in advanced imaging, particularly in oncology, metabolic disorders and inflammation. Through this interview, Fournal of Thoracic Disease (FTD) is interested to know the critical issues facing the field, some latest advances in advanced imaging, the effectiveness of computed tomography (CT) in lung cancer screening, the recent research projects that Prof. Chaudhry is currently working on, as well as the interesting and challenging aspects during research and the pursuit of his lifelong mission—the realization of "virtual biopsy".

\section{Expert introduction}

Prof. Ammar Ahmed Chaudhry, MD, currently serves as Assistant Clinical Professor of Diagnostic Radiology at City of Hope, Duarte, California, the US (Figure 1). After receiving his medical degree with honors from University of South Florida College of Medicine, he completed residency at Stony Brook University Medical Center and then his fellowship at Johns Hopkins School of Medicine, where he stayed on as faculty before joining City of Hope.

As the founder and director of the Precision Imaging Lab at City of Hope, Prof. Chaudhry's research has been centered on applications of advanced imaging [functional magnetic resonance imaging ( $\mathrm{fMRI}$ ), positron emission tomography-MRI (PET-MRI), perfusion weighting imaging (PWI), hyperpolarized MRI] in oncology,

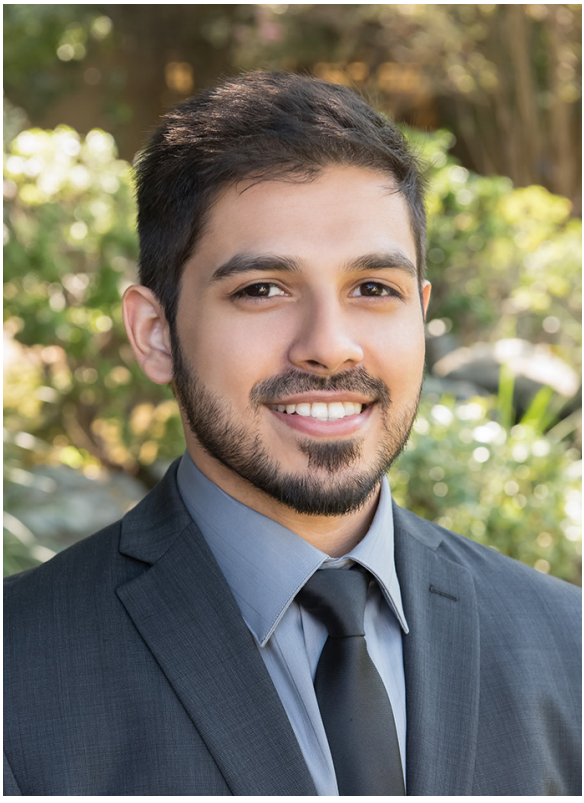

Figure 1 Prof. Ammar Ahmed Chaudhry.

metabolic disorders and inflammation. His goal is to expand the options for non-invasive diagnosis and treatment of cancer and other diseases. He is actively involved in a wide range of academic activities-publishing hundreds of research articles, presentations and abstracts, reviewing for several peer-reviewed scientific journals, and giving lectures at different national/international scientific conventions. He earned several teaching awards and was presented the Young Investigator Award by City of Hope in 2017.

\section{Interview}

FTD: Having been in the field of advanced imaging in oncology, metabolic disorders and inflammation for years, what do you think are the critical issues facing the field right now?

Prof. Chaudhry: One of the main issues is lack of specificity in advanced imaging. Conventional CT, PET- 
CT, and MRI are good in their respective sensitivities of detecting a lesion. However, the lack of specificity makes diagnosing challenging. This is further compounded by factors such as increasing incidence of multiple cancers in patients (e.g., breast and lung cancer, lymphoma and lung cancer, melanoma and lung lesions). In the aforementioned cases, it is difficult to ascertain whether the lung lesions are primary lung or metastatic (especially if there is one lung nodule).

Cost and availability are another critical issue in advanced imaging. Advanced imaging is costly- partially because of the time commitment but also because emerging technologies carry the burden of innovation cost. As such, patients generally have limited access to these technologies-usually at academic centers \pm clinical trials set up. Finding creative ways to budget these emerging advanced imaging techniques presents a unique challenge.

\section{FTD: What are some latest advances in the field of advanced imaging in the recent decade?}

Prof. Chaudhry: PET-MRI-this "two-in-one" modality combines the strength of PET and MRI and allows for simultaneous acquisition of PET and MRI sequences. Benefits include: (I) time efficient-instead of getting a separate MRI and PET exam (can be up to two hours), patient undergoes simultaneous PET-MRI which can perform whole body MRI and PET series acquisitions within one hour; (II) MRI allows for improved tissue contrast, improved spatial resolution, without the burden of ionizing radiation from CT (as is the case in PET/CT). PET adds functional information which compliments MRI findings, thus allowing for more precise evaluations of lesions.

ImmunoPET (iPET)—instead of using conventional FDG (18-fluorodeoxyglucose) as radiotracer, iPET works via radioconjugation of specific antibodies and/or small molecules that target cell surface receptors. This provides a targeted approach to tumor cell detection. For example, ${ }^{89} \mathrm{Zr}$-Girentuximab iPET is radioconjugate Girentuximab with zirconium, which is a carbonic anhydrase IX (CAIX) inhibitor over expressed in clear cell renal cell carcinoma (ccRCC).

Chemical exchange saturation transfer (CEST) - MRI is a technique which can detect compounds that have exchangeable component (e.g., proton, amide, etc.). This technique takes advantage of the magnetization transfer of mobile compounds (e.g., water, amino acids, etc.). CEST evaluates slow exchange (proton, amide, etc.) over time and this imaging techniques gain signal from both the direct chemical exchange as well as through dipolar crossrelaxation. This emerging technique can potentially be used to evaluate tumor microenvironment and metabolomics.

\section{FTD: What role does CT play in lung cancer management? What are the limitations of the use of CT in lung cancer screening?}

Prof. Chaudhry: CT is currently the primary diagnostic tool being used for primary lung cancer detection and posttreatment follow-up. CT provides the optimal spatial and temporal contrast resolution required to evaluate pulmonary lesions, especially at an early ('in situ') stage where these lesions are generally less than $10 \mathrm{~mm}$. CT can readily detect lesions from 0.3 to $0.5 \mathrm{~mm}$ (in some of the newer CT scanners) to $1 \mathrm{~mm}$ and above. Artifact from air and motion has limited utility of MRI in diagnosis and management of primary lung cancer. PET, due to its spatial resolution limitations, works well when combined with CT (PET-CT).

Although CT is excellent in its sensitivity of detecting lung lesions, there are a few limitations. The biggest limitation is the lack of specificity, especially when lesion morphologically is well defined. In such scenarios, if lesion is 'sizeable' ( $>7 \mathrm{~mm}$ ), then PET can potentially identify metabolic activity which can provide to additional pathologic or benign characteristics of the detected nodule. However, if there is minimal to mild or no PET activity, then in patients with risk factors (e.g., age, smoking, nodule size, etc.) the nodule is either followed-up over serial CT's or biopsied. Also, lung cancer screening becomes more complex in patients with lung cancer risk factors who have history of additional malignancies (e.g., colon cancer, smoking related cancers, renal, bladder, pancreatic cancers, etc.). In such subjects, it is sometimes difficult to ascertain whether the lung lesion is primary or secondary neoplasm and often requires confirmatory biopsies.

\section{FTD: Would you introduce us to the National Institutes of Health (NIH)-funded project, namely "City of Hope Clinical Oncology Career Research Development Program"?}

Prof. Chaudhry: City of Hope Clinical Oncology Career Research Development program is designed for young investigators, such as myself, with interest in improving patient care through research and discovery. The program 
started in 1991 and is funded by the NIH. Dr. Joanne Mortimer is the institutional Principal Investigator who oversees the research scholars. The objective of the award is to "develop the next generation of academic oncologists in a variety of multidisciplinary fields with an ability to translate advances in cancer biology into novel strategies for the diagnosis, therapy and prevention of malignant disease.

The program is highly competitive and candidates are selected based on various criteria. The program provides a multidisciplinary curriculum including didactics, along with clinical experience and laboratory in basic, translational and clinical research within cancer-related fields. All the scholars have two mentors: one clinical and one basic science, both with excellent track record of innovative, funded research. I am fortunate to have Dr. Behnam Badie (Neurosurgery) and Dr. Andrea Bild (Pharmacology) as my two mentors. Under their mentorship, I have developed my Precision Imaging Lab where I am performing both diagnostic and therapeutic research testing our scientific hypotheses. At present, I am in phase I of my grant where I'm developing novel imaging agents and coupling those with the aforementioned emerging advanced imaging technologies (PET-MRI, iPET, CEST-MRI).

\section{FTD: Your goal is to expand the options for non-invasive diagnosis and treatment of cancer and other diseases. To what extent have you fulfilled your goal so far?}

Prof. Chaudhry: As my goal is to expand options for noninvasive diagnosis and treatment, in phase I of my research, I have developed iPET agents and MRI contrast agents. We are conducting in vitro studies to develop the model that we use for our phase II (in vivo) imaging. Early in vitro findings are promising and we are hoping to start our phase II in vivo imaging in the last quarter of 2018.

\section{FTD: What do you regard as the most interesting aspects of research? What have been driving you to move forward to achieve your goal?}

Prof. Chaudhry: One of the most interesting aspects of research is compiling the present knowledge on a disease, identifying frontiers where highest impact research can be conducted and generating hypotheses. Data analysis is another interesting component of research not only because that is where one identifies whether the hypothesis was validated or not, but also some of the data raises questions that pave way for the next round of research projects. Lastly, as one's career matures, seeing the body of your work answer questions and direct research within and outside your own institutional is exciting and fulfilling.

Not to be cliché', but the primary drivers of my research have been my parents. I became interested in medicine when my father had a heart attack when I was 8 years old. As I accompanied my father to his appointments, I heard various medical terminologies that I had no idea about, but I was interested in knowing what they meant-not just their definition, but what it meant prognostically for my father. When I was 13, my mother was diagnosed with pulmonary fibrosis secondary to her scleroderma. The final diagnosis of pulmonary fibrosis was made after my mother had gone through a series of biopsies. Seeing her go through the challenges of complicated biopsies made me want and hope for "virtual biopsy" and noninvasive therapies. Upon discovering, there is no such thing as "virtual biopsy", I took it upon myself as my life's mission to develop it. That is what leads me to diagnostic and interventional radiology, which is the field where I felt I could pioneer "virtual biopsy".

FTD: As the founder and director of the Precision Imaging Lab at City of Hope, were there any obstacles when you first started it up?

Prof. Chaudhry: Translating the concept of "virtual biopsy" into reality has not been an easy task. As one would expect, hearing a teenager talk about "virtual biopsy" and noninvasive therapies was taken up with a lot of cynicism and ridicule. Early on, I realized that turning a concept into something tangible is always hard. Precision Imaging Lab started with the idea of "virtual biopsy". There have been many obstacles which are financial, geospatial as well as other miscellaneous ones. I have been able to overcome financial challenges through grant funding (NIH and foundational grants as well as institutional support). The financial support almost directly correlates with degree of academic success.

One of my most memorable moments was my publication on Characteristics CT Findings of Cryoablation Treatment of Malignant Lung Nodules (PMID 26496275). First, the paper showed our clinical outcomes after cryotherapy were comparable to external control (patient who have undergone surgical resection). Interestingly, our patient population was older and had a poorer functional score than the surgical cohort. Additionally, our paper defined the imaging criteria to use when evaluating post-cryoablation zones in the lungs. 
This was the first systematically studies hypothesis driven publication in this cohort. This publication was important, because it gave me the evidence I needed to present to the institutional leadership in order to get Precision Imaging Lab started and move forward with my mission of "virtual biopsy".

\section{FTD: What are the key factors of a successful research in your opinion?}

Prof. Chaudhry: There are several key factors. The most important I believe is "doing the right thing for the right reasons". I think if the investigator is passionate, honest, hardworking and driven to make positive impact, success will come. Another key factor is defining success. To me "success" is not a "positive" result of an experiment. Success is executing the entire experiment using the scientific method and accruing and analyzing results as they become

Cite this article as: Li B. Ammar Ahmed Chaudhry: do the right thing for the right reasons! J Thorac Dis 2018;10(11):E787-E790. doi: $10.21037 /$ jtd.2018.10.18 available. Cumulative success in completing the experiment will help one in redefining and improving standard of care. This, my hope is, will bring the "ultimate success" which will be making "virtual biopsy" a reality for the patients and not just a figment of my imagination.

\section{Acknowledgements}

We would like to express our sincerest gratitude to Prof. Ammar Ahmed Chaudhry for sharing his insights and opinions with us.

\section{Footnote}

Conflicts of Interest: The author has no conflicts of interest to declare.

(Science Editor: Brad Li, JTD, jtd@amepc.org) 